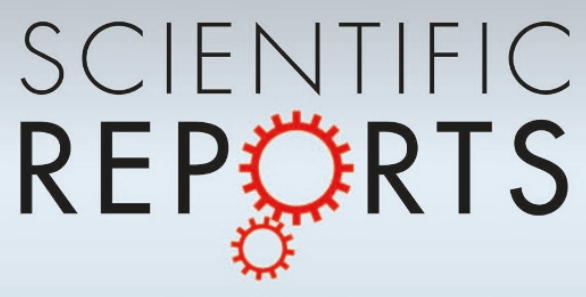

OPEN

SUBJECT AREAS:

STRUCTURAL PROPERTIES

ELECTRONIC PROPERTIES AND

MATERIALS

Received

10 June 2014

Accepted

1 August 2014

Published

22 August 2014

Correspondence and requests for materials should be addressed to S.R. (shenqiang@ku. edu)

\section{Charge-Transfer Induced Magnetic Field Effects of Nano-Carbon Heterojunctions}

\author{
Wei Qin', Maogang Gong', Tejas Shastry², Mark C. Hersam² \& Shenqiang Ren'
}

'Department of Chemistry, University of Kansas, Lawrence, KS 66045, United States, 2 Department of Materials Science and
Engineering and Department of Chemistry, Northwestern University, Evanston, IL 60208, United States.

Room temperature magnetic field effects have not been definitively observed in either single-walled carbon nanotubes (SWCNTs) or $\mathrm{C}_{60}$ under a small magnetic field due to their weak hyperfine interaction and slight difference of $g$-factor between positive and negative polarons. Here, we demonstrate charge-transfer induced magnetic field effects in nano-carbon $\mathrm{C}_{60}$-SWCNT bulk heterojunctions at room temperature, where the mechanism of magnetic field effects is verified using excited state transition modeling. By controlling SWCNT concentrations and interfacial interactions, nano-carbon heterojunctions exhibit tunability of charge-transfer density and room temperature magnetoconductance of $2.8 \%$ under $100 \mathrm{mT}$ external magnetic field. External stimuli, such as electric field and photoexcitation, also play an important role in controlling the magnetic field effects of nano-carbon heterojunctions, which suggests that these findings could enable the control of optoelectronic properties of nano-carbon heterojunctions.

M agnetic field effects (MFE) under small magnetic field (tens of millitesla) in organic semiconductors are extremely attractive due to their combined electronic, optical, and magnetic characteristics for data storage, optoelectronics, and sensing ${ }^{1,2}$. To understand the mechanism of MFE, a large number of organic systems have been investigated ${ }^{3-9}$, where the bipolaron mechanism ${ }^{10,11}$, polaron pair mechanism ${ }^{12,13}$ and triplet-polaron interaction mechanism ${ }^{14,15}$ have been proposed. All these mechanisms rely on spin-selective reactions between pairs, where a magnetic field can tune the degree of spin mixing. Compared to the MFE in small molecules and polymers, the $\mathrm{sp}^{2}$-carbon allotropes (such as fullerenes or carbon nanotubes) have shown extremely weak MFE at room temperature. For example, MFE of single walled carbon nanotubes (SWCNTs) can only be observed with tens of tesla magnetic field at extremely low temperature ${ }^{16,17}$. The sp $\mathrm{p}^{2}$-carbon consists of $1.1 \%{ }^{13} \mathrm{C}$ isotope (spin 1/2) and $98.9 \%{ }^{12} \mathrm{C}$ isotope (no spin), which lacks hyperfine interaction ${ }^{18}$. Wohlgenannt et al concluded that the rather weak hyperfine interaction is responsible for the extremely low MFE in these nanocarbon materials ${ }^{19}$. However, enhancing the hyperfine interaction through increasing ${ }^{13} \mathrm{C}$ isotope concentration $\left(25 \%{ }^{13} \mathrm{C}\right.$ isotope) in the $\mathrm{C}_{60}$ system did not show an improvement of $\mathrm{MFE}^{18}$. Therefore, tuning the strength of hyperfine interaction is not an effective way to enhance MFE in $\mathrm{sp}^{2}$-carbon allotropes.

In this study, we show for the first time that a nano-carbon bulk heterojunction consisting of $\mathrm{C}_{60}$ (electron acceptor) and semiconducting SWCNTs (electron donor) exhibits charge-transfer induced MFE. The density of charge-transfer states within nano-carbon heterojunctions can be tuned by controlling the SWCNT concentration and applied electric field, leading to charge-transfer dependent MFE. By taking into account the Zeeman interaction, hyperfine interaction and exchange interaction of charge-transfer, we build a model to understand the mechanism of MFE in nano-carbon heterojunctions devices through calculation of magnetic field dependent singlet and triplet ratios. A $1.9 \%$ magnetoconductance (MC) is achieved under $3 \mathrm{wt} \%$ SWCNT loading and $5 \mathrm{MV} / \mathrm{m}$ electric field at room temperature, a magnitude that is much larger than that of previous $\mathrm{C}_{60}$-based devices $^{17,18}$ and similar to other organic systems. In addition, increasing interfacial interactions by controlling the morphology and interfacial area further enhances the $\mathrm{MC}$ to $2.8 \%$ at room temperature.

\section{Results}

The nano-carbon bulk heterojunction consists of a blend of $\mathrm{C}_{60}$ and SWCNTs. The broad-spectral photoabsorption from the nano-carbon heterojunction at both visible and near-infrared (NIR, Figure 1a) wavelengths creates opportunities for the formation of photoexcited charge-transfer states. From transmission electron microscopy (TEM) images, it is noted that the nano-carbon heterojunction exhibits a random-mixture morphology, as shown in Figure 1b. It should be noted that current density becomes larger when applying a magnetic field in $\mathrm{C}_{60^{-}}$ SWCNT heterojunction devices, leading to the positive $\mathrm{MC}(\mathrm{MC}$ is defined as: $\mathrm{MC}=[j(\mathrm{~B})-j(0)] / j(0), j(\mathrm{~B})$ is 

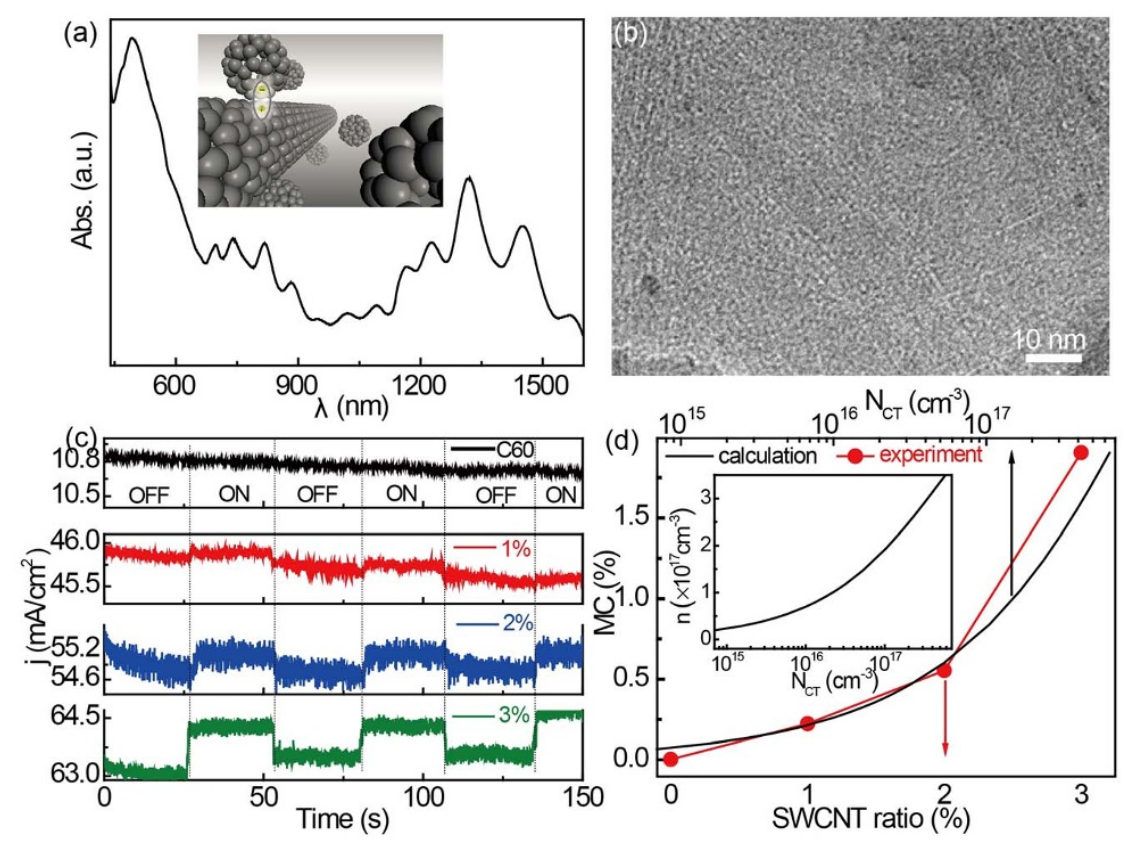

Figure 1 The photoabsorption of the $\mathrm{C}_{60}-\mathrm{SWCNT}$ nano-carbon (a), the inset of (a) shows the scheme of the $\mathrm{C}_{60}-\mathrm{SWCNT}$ nano-carbon heterojunction; TEM image of a $\mathrm{C}_{60}$-SWCNT nano-carbon heterojunction, (b) Magnetic field effect in $\mathrm{C}_{60}$-SWCNT heterojunctions with 1 wt $\%$, 2 wt $\%$ and 3 wt $\%$ SWCNT (c), OFF (ON) means magnetic field is tuned off (on), and $B=100 \mathrm{mT}$. Theoretical calculation (black line) compared with the experimental result (red circle) (d).

current density of the device with magnetic field) value shown in Figures $1 \mathrm{c}$ and $1 \mathrm{~d}$. The applied magnetic field could induce less spin mixing, which can leads to more triplet charge-transfer ${ }^{20,21}$. Triplet charge-transfers can further translate into triplet excitons which can also react with free charge carriers due to their long lifetime ${ }^{22,23}$. The relevant outcome of this type of reaction is the electron and hole separation of triplet exciton ${ }^{24,25}$, which induces larger carrier density and thus a positive MC. By tuning magnetic field $(100 \mathrm{mT})$ on/off, it is found that the current density of $\mathrm{C}_{60}-\mathrm{SWCNT}$ nano-carbon devices exhibits the same responsive on/off behavior at room temperature.

To understand the mechanism of charge-transfer dependent magnetic field effects, theoretical dynamic transition equation ${ }^{21}$ among electrons, holes, charge-transfers and excitons are built as,

$$
\begin{aligned}
\frac{d n_{-}\left(n_{+}\right)}{d t}= & -\beta_{L} n_{-} n_{+}+k_{D}(E) N_{C T-S}+k_{D}(E) N_{C T-T}+k_{T} N_{E X-T} \\
\frac{d N_{C T-S}}{d t}= & \beta_{L} \frac{1}{4} n_{-} n_{+}-\gamma_{S} N_{C T-S}+\gamma_{T} N_{C T-T}-k_{D}(E) N_{C T-S} \\
& -\alpha_{S 1} N_{C T-S}+\alpha_{S 2} N_{E X-S} \\
\frac{d N_{C T-T}}{d t}= & \beta_{L} \frac{3}{4} n_{-} n_{+}-\gamma_{T} N_{C T-T}+\gamma_{S} N_{C T-S}-k_{D}(E) N_{C T-T} \\
& -\alpha_{T 1} N_{C T-T}+\alpha_{T 2} N_{E X-T} \\
\frac{d N_{E X-S}}{d t}= & \alpha_{S 1} N_{C T-S}-\alpha_{S 2} N_{E X-S} \\
\frac{d N_{E X-T}}{d t}= & \alpha_{T 1} N_{C T-T}-\alpha_{T 2} N_{E X-T}-k_{T} N_{E X-T}
\end{aligned}
$$

Electron (hole) density $n_{-}\left(n_{+}\right)$is determined by three parts: recombination $\left(\beta_{L}\right)$, dissociation of charge-transfers $\left(k_{D}\right)$ and triplet exciton-free charge interaction induced triplet exciton dissociation $\left(k_{T}\right)^{9,24,25}$. The recombination coefficient is $\beta_{L}=e\left(\mu_{-}+\mu_{+}\right) / \varepsilon_{0} \varepsilon_{r}$ and dissociation rate is $k_{D}(E)=\frac{3<\mu>e}{4 \pi \varepsilon_{0} \varepsilon_{r} d^{2}} \exp \left(-\frac{R_{C}}{d}\right) \frac{J_{1}(2 \sqrt{-2 b E})}{\sqrt{-2 b E}}$, where $J_{1}$ is the one order Bessel function and $b=e^{3} / 8 \pi \varepsilon_{0} \varepsilon_{r} k_{B}^{2} T^{2}, d$ corresponds to a electron-hole distance of charge-transfers. $R_{\mathrm{C}}$ is the Coulomb capture radius, $E$ is electric field, $\varepsilon_{r}$ the dielectric constant and parameters are: $B_{\mathrm{hf}}=a / g \mu_{\mathrm{B}}=0.1 \mathrm{mT}, B_{\mathrm{J}}=J / g \mu_{\mathrm{B}}=10 \mathrm{mT}^{21}, d$ $=1.3 \mathrm{~nm}^{26}, R_{\mathrm{C}}=4 \mathrm{~nm}^{27} \cdot \gamma_{S}$ and $\gamma_{T}$ in the equation indicate the mutual conversion between singlet $\left(N_{C T-S}\right)$ and triplet $\left(N_{C T-T}\right)$ charge-transfers, which are determined by the magnetic field (the expressions of $\gamma_{S}$ and $\gamma_{T}$ can be obtained by solving the time dependent Schrodinger Equation ${ }^{21}$ ). The transition rate from triplet chargetransfers to singlet ones is expressed as $\gamma_{T}=$ $\frac{1}{16}\left[4-\frac{\left(\omega^{2}+\frac{1}{2} J^{2}+\omega J\right)^{2}}{4\left(a^{2}+\omega^{2}+\frac{1}{2} J^{2}+\omega J\right)^{2}}-\frac{\left(\omega^{2}+\frac{1}{2} J^{2}-\omega J\right)^{2}}{4\left(a^{2}+\omega^{2}+\frac{1}{2} J^{2}-\omega J\right)^{2}}\right] \gamma_{0}, \quad$ and the rate from singlet charge-transfers to triplet ones is expressed as $\gamma_{S}=\frac{1}{16}\left[12+\frac{\left(\omega^{2}+\frac{1}{2} J^{2}+\omega J\right)^{2}}{4\left(a^{2}+\omega^{2}+\frac{1}{2} J^{2}+\omega J\right)^{2}}+\frac{\left(\omega^{2}+\frac{1}{2} J^{2}-\omega J\right)^{2}}{4\left(a^{2}+\omega^{2}+\frac{1}{2} J^{2}-\omega J\right)^{2}}\right] \gamma_{0}$, where $\omega=g \mu_{B} B$ is a parameter which is related to external magnetic field $B$, $a$ means hyperfine interaction strength and $J$ is exchange interaction strength of charge-transfer. Thus, the magnetic field can tune the ratios of singlet and triplet charge-transfers. Singlet and triplet exciton densities are $N_{E X-S}$ and $N_{E X-T}$, respectively.

By solving equation (1), the magnetic field dependent carrier density can be obtained. The current density, $j=n_{+} e \mu_{+} E+n_{-} e \mu_{-} E$, can be tuned by the magnetic field, which induces magnetic field effects in $\mathrm{C}_{60}$-SWCNT nano-carbon heterojunctions. The carrier density $n$ improves with increasing charge-transfer density $N_{\mathrm{CT}}$, as shown in the inset of Figure 1d. The magnetic field effect becomes more apparent by increasing SWCNT loading ratio, and the MC value can reach as high as $1.9 \%$ with $3 \mathrm{wt} \%$ SWCNT loading ratio, as shown in Figure 1d. At loadings higher than $3 \mathrm{wt} \%$, the devices become short due to the poor dispersibility of SWCNTs ${ }^{28}$. The MFE in $\mathrm{C}_{60}$-SWCNT nano-carbon devices is significantly larger than that of previous $\mathrm{C}_{60}$-based devices $\left(\mathrm{MC}=0^{19}\right.$ and $\mathrm{MC}=0.02 \%{ }^{18}$ ) when the applied magnetic field is $100 \mathrm{mT}$. In addition, as shown in Figure 1d, our calculation on charge-transfer density dependent MC matches the experimental data well.

To further study the charge-transfer dependent $\mathrm{MC}$ in $\mathrm{C}_{60^{-}}$ SWCNT nano-carbon device, we apply different electric field strengths and light intensities to control MFE, as shown in 

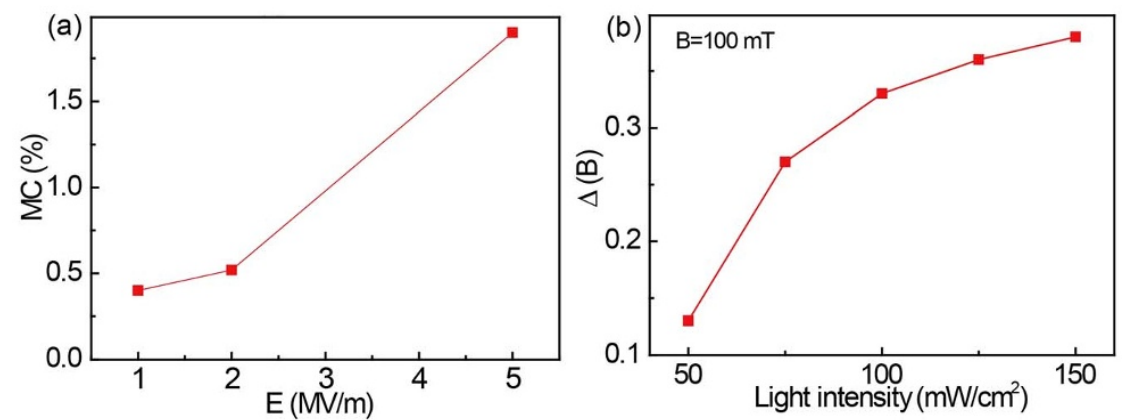

Figure $2 \mid$ The tunability of magnetic field effect by electric field (a) and light intensity, (b) The SWCNT loading ratio is 3 wt\% in this case.

Figures $2 \mathrm{a}$ and $2 \mathrm{~b}$. By increasing applied electric field, more electrons and holes are injected into the $\mathrm{C}_{60}$-SWCNT nano-carbon active layer. If the distance between electron and hole is smaller than a critical value, the electron and hole will be combined together by Coulomb attraction to form charge-transfer state. In particular, Onsager ${ }^{27,29}$ proposed a definition for a Coulomb capture radius $r$ at which the Coulomb attraction energy equals the thermal energy $k_{B} T: r=\frac{e^{2}}{4 \pi \varepsilon_{0} \varepsilon_{r} k_{B} T}, e$ is the elementary charge, $\varepsilon_{r}$ is the dielectric constant of the surrounding medium, $\varepsilon_{0}$ permittivity of vacuum. The Coulomb attraction radius emphasizes the importance of the dielectric constant of the materials. Due to the small dielectric constant of nano-carbon $\left(\varepsilon_{r}<6\right)^{30,31}$, the Coulomb capture radius is large, and charge-transfer states bound by Coulomb attraction are produced
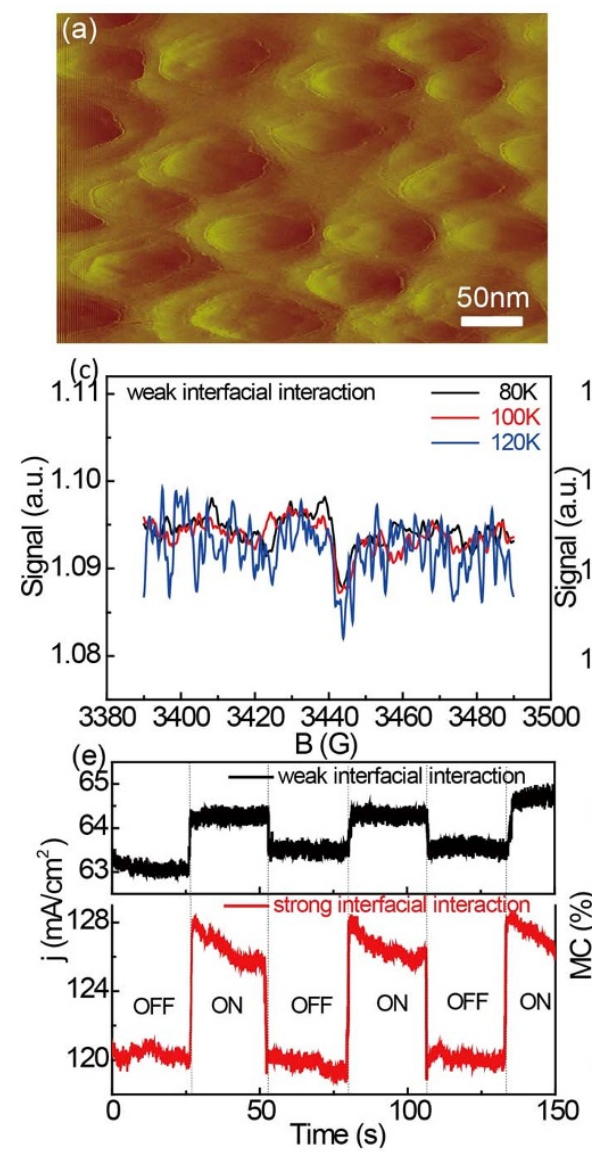

with high efficiency. Increasing electron and hole densities will contribute to the formation of a large density of charge-transfer states. Therefore, the MC effect becomes more apparent with a larger electric field, as shown in Figure 2a. In addition to electric field controlled charge-transfer density in $\mathrm{C}_{60}$-SWCNT nano-carbon devices, photoexcitation is also an effective method to excite a large number of charge-transfer states. To measure magnetic field dependent light response in $\mathrm{C}_{60}$-SWCNT nano-carbon devices, we define the optical switch value as: $j_{D}=j$ (on) $-j$ (off), where $j$ (on) and $j$ (off) are electric current densities with and without light illumination, respectively. Figure $2 \mathrm{~b}$ shows the magnetic field dependent light response: $\Delta(\mathrm{B})=\frac{j_{D}(\mathrm{~B})-j_{D}(0)}{j_{D}(0)}$ under different light intensity and broad-spectrum illumination, where $j_{D}(0)=j($ on, $\mathrm{B}=0)-j($ off, $\mathrm{B}=0)$ is the
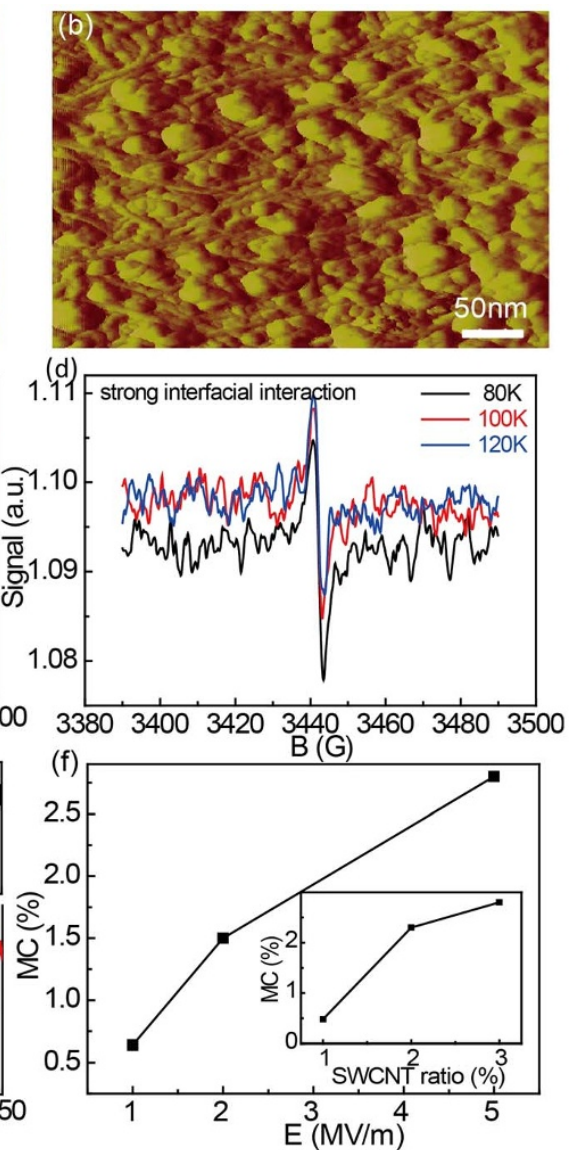

Figure 3 Atomic force microscopy image of $\mathrm{C}_{60}$-SWCNT blends with weak interfacial interaction (a) and strong interfacial interaction, (b) ESR signal with $1 \%$ SWCNT loading ratio in weak interfacial interaction nano-carbon heterojunctions (c) and strong interfacial interaction nano-carbon heterojunctions (d). Magnetic field effects with strong and weak interfacial interaction (e), OFF (ON) means magnetic field is tuned off (on). (f) Electric field (SWCNT ratio is $3 \mathrm{wt} \%$ ) and SWCNT ratio dependent MC in strong interfacial interaction nano-carbon heterojunctions. 
light response without applying magnetic field, and $j_{D}(\mathrm{~B})=j($ on, $\mathrm{B})$ $-j($ off, $B)$ means the light response under the effect of magnetic field. It is noted that optical switch effect becomes more apparent once applying magnetic field, leading to a positive $\Delta(\mathrm{B})$. By further increasing light intensity, more charge-transfer states are excited to enhance the MFE.

As presented above, the charge-transfer states in $\mathrm{C}_{60}-\mathrm{SWCNT}$ nano-carbon heterojunctions play an important role in magnetic field effects. In addition, the morphology and interfacial interactions of nano-carbon heterojunctions dictate their charge-transfer process. Therefore, we utilized electron spin resonance (ESR) to further understand the effect of phase separated nano-carbon heterojunctions on charge-transfer induced magnetic field effects. The surface aggregation of $\mathrm{C}_{60}$ and phase segregation with top $\mathrm{C}_{60}$-rich layer is clearly present in the surface morphology of nano-carbon heterojunctions without extended sonication during the $\mathrm{C}_{60}$ and SWCNT blending process (Figure 3a). It should be noted that SWCNTs tend to aggregate in solution to form bundles, which limit the interfacial interaction between SWCNT and $\mathrm{C}_{60}$ within nano-carbon bulk heterojunctions. However, a uniform surface morphology with both $\mathrm{C}_{60}$ and SWCNT is observed after sonication (Figure 3b). The weak ESR signal of nano-carbon heterojunctions without sonication disappeared above $120 \mathrm{~K}$ due to limited interfacial interaction [Fig. 3(c)]. Therefore, uniform nano-carbon heterojunction morphology after sonication could enhance the interfacial interaction between $\mathrm{C}_{60}$ and SWCNTs (Figure 3b), leading to a drastically enhanced ESR signal (Figure 3d). Upon increasing the SWCNT ratio to $2 \mathrm{wt} \%$ and $3 \mathrm{wt} \%$, the ESR signal is more apparent (Supplementary Information, Figure S4). Temperature dependent ESR signals with different SWCNT ratios are presented in Supplemental Material (Figure S5). It is found that strengthening interfacial interaction by sonication-induced nano-carbon heterojunction could enhance MC (Figure 3e), compared to a low response of the phase segregated one. In addition, the MC effect becomes more apparent with increasing electric field or SWCNT loading ratios on the sonication-processed nano-carbon heterojunctions, as shown in Figure 3f. In particular, by loading $3 \mathrm{wt} \%$ SWCNT in $\mathrm{C}_{60}$-SWCNT nano-carbon device, the $\mathrm{MC}$ can reach as high as $2.8 \%$ at room temperature under $100 \mathrm{mT}$ applied magnetic field.

\section{Discussion}

Charge-transfer dependent magnetic field effects are presented for the first time in $\mathrm{C}_{60}$-SWCNT nano-carbon heterojunctions. The singlet and triplet ratios of $\mathrm{C}_{60}$-SWCNT nano-carbon heterojunctions can be tuned by external magnetic field, leading to pronounced magnetic field effects. By tuning charge-transfer density through control of the SWCNT loading ratios, $1.9 \%$ of magnetoconductance is achieved with $3 \mathrm{wt} \%$ SWCNT loading ratio under $100 \mathrm{mT}$ magnetic field at room temperature, in agreement with theoretical calculations. To further investigate the effect of charge-transfer on magnetic field effects, electric field and light illumination are used to control charge-transfer density in nano-carbon heterojunctions. In addition, by increasing interfacial interactions of $\mathrm{C}_{60}$-SWCNT nano-carbon heterojunctions through morphology and phase separation control, the magnetoconductance is further improved to $2.8 \%$ under $100 \mathrm{mT}$ magnetic field at room temperature.

\section{Method}

Device structure. Indium tin oxide (ITO) was chosen as the transparent bottom electrode. After cleaning the ITO substrate, Poly(3,4-ethylenedioxythiophene) Polystyrene sulfonate (PEDOT:PSS) was coated at $3500 \mathrm{rpm}$ for 1 minute. Semiconducting SWCNTs were prepared by density gradient ultracentrifugation from raw HiPCo as previously described ${ }^{32}$. The resulting aqueous dispersions were characterized for semiconducting purity. Surfactants were removed from the SWCNTs by flocculating in ethanol, filtering onto a nylon membrane, and rinsing the membrane in acetone and IPA ${ }^{33}$. The resulting dry film was weighed and retained for later use. The active layer consists of $\mathrm{C}_{60}$-SWCNT (the concentration of the $\mathrm{C}_{60}$ solution is $30 \mathrm{mg} / \mathrm{mL}$ in 1,2-Dichlorobenzene and the SWCNT concentration is
$2 \mathrm{mg} / \mathrm{mL}$ ), which was spun-cast at $1000 \mathrm{rpm}$ for 1 minute. Aluminum was chosen as the top electrode through thermal evaporation. The device area was defined as $0.65 \times$ $1.75 \mathrm{~mm}^{2}$.

Photoabsorption. A Shimadzu UV-3600 spectrophotometer was used to measure the absorption spectrum of the $\mathrm{C}_{60}$-SWCNT nano-carbon heterojunction.

Low temperature ESR measurement. The mixed $\mathrm{C}_{60}$ and SWCNT solution was stirred for 10 hours at room temperature (SWCNT ratios are $1 \mathrm{wt} \%, 2 \mathrm{wt} \%$ and $3 \mathrm{wt} \%$ ). $150 \mu \mathrm{L}$ of the $\mathrm{C}_{60}$-SWCNT solution was injected into the glass tube under $\mathrm{N}_{2}$ environment. The low temperature ESR measurement was done under the average of 10 sweeps.

j-V measurement. A Keithley 2400 source generator was used to measure $\mathrm{j}-\mathrm{V}$ of the $\mathrm{C}_{60}$-SWCNT heterojunction devices in a nitrogen atmosphere glovebox.

1. Baker, W. J. et al. Robust absolute magnetometry with organic thin-film devices. Nat. Commun. 3, 898 (2012).

2. Raman, K. V. et al. Interface-engineered templates for molecular spin memory devices. Nature 493, 509-513 (2013).

3. Bloom, F. L., Wagemans, W., Kemerink, M. \& Koopmans, B. Separating positive and negative magnetoresistance in organic semiconductor devices. Phys. Rev. Lett. 99, 257201 (2007).

4. Bloom, F. L., Wagemans, W., Kemerink, M. \& Koopmans, B. Correspondence of the sign change in organic magnetoresistance with the onset of bipolar charge transport. Appl. Phys. Lett. 93, 263302 (2008).

5. Nguyen, T. D., Gautam, B. R., Ehrenfreund, E. \& Vardeny, Z. V. Magnetoconductance response in unipolar and bipolar organic diodes at ultrasmall fields. Phys. Rev. Lett. 105, 166804 (2010).

6. Francis, T. L., Mermer, Ö., Veeraraghavan, G. \& Wohlgenannt, M. Large magnetoresistance at room temperature in semiconducting polymer sandwich devices. New J. Phys. 6, 185 (2004).

7. Wang, F. J., Bässler, H. \& Vardeny, Z. V. Magnetic field effects in $\pi$-conjugated polymer-fullerene blends: evidence for multiple components. Phys. Rev. Lett. 101, 236805 (2008).

8. Xie, Y. et al. Broad-spectral-response nanocarbon bulk-heterojunction excitonic photodetectors. Adv. Mater. 25, 3433-3437 (2013).

9. Hu, B., Yan, L. \& Shao, M. Magnetic-field effects in organic semiconducting materials and devices. Adv. Mater. 21, 1500-1516 (2009).

10. Wagemans, W., Bloom, F. L., Bobbert, P. A., Wohlgenannt, M. \& Koopmans, B. A two-site bipolaron model for organic magnetoresistance. J. Appl. Phys. 103, 07F303 (2008).

11. Bobbert, P. A., Nguyen, T. D., Van-Oost, F. W. A., Koopmans, B. \& Wohlgenannt, M. Bipolaron mechanism for organic magnetoresistance. Phys. Rev. Lett. 99, 216801 (2007).

12. Majumdar, S. et al. Role of electron-hole pair formation in organic magnetoresistance. Phys. Rev. B 79, 201202 (2009).

13. Bagnich, S. A., Niedermeier, U., Melzer, C., Sarfert, W. \& Seggern, H. V. Electronhole pair mechanism for the magnetic field effect in organic light emitting diodes based on poly(paraphenylene vinylene). J. Appl. Phys. 106, 113702 (2009).

14. Desai, P. et al. Magnetoresistance and efficiency measurements of $\mathrm{Alq}_{3}$ based OLEDs. Phys. Rev. B 75, 094423 (2007).

15. Desai, P., Shakya, P., Kreouzis, T. \& Gillin, W. P. Magnetoresistance in organic light-emitting diode structures under illumination. Phys. Rev. B 76, 235202 (2007).

16. Kim, G. T. et al. Magnetoresistance of an entangled single-wall carbon-nanotube network. Phys. Rev. B 58, 16064-16069 (1998).

17. Fedorov, G. et al. Magnetically induced field effect in carbon nanotube devices. Nano Lett. 7, 960-964 (2007).

18. Gautam, B. R., Nguyen, T. D., Ehrenfreund, E. \& Vardeny, Z. V. Magnetic field effect spectroscopy of $\mathrm{C}_{60}$-based films and devices. J. Appl. Phys. 113, 143102 (2013).

19. Nguyen, T. D., Sheng, Y., Wohlgenannt, M. \& Anthopoulos, T. D. On the role of hydrogen in organic magnetoresistance: A study of $\mathrm{C}_{60}$ devices. Synth. Met. 157, 930-934 (2007).

20. Janssen, P. et al. Tuning organic magnetoresistance in polymer-fullerene blends by controlling spin reaction pathways. Nat. Commun. 4, 2286 (2013).

21. Qin, W., Gao, K., Yin, S. \& Xie, S. J. Investigating the magnetic field effect on electron-hole pair in organic semiconductor devices. J. Appl. Phys. 113, 193901 (2013).

22. Baldo, M. A. et al. Highly efficient phosphorescent emission from organic electroluminescent devices. Nature 395, 151-154 (1998).

23. Wang, F., Dukovic, G., Brus, L. E. \& Heinz, T. F. Time-resolved fluorescence of carbon nanotubes and its implication for radiative lifetimes. Phys. Rev. Lett. 92, 177401 (2004).

24. Wittmer, M. \& Zschokke-Gränacher, I. Exciton-charge carrier interactions in the electroluminescence of crystalline anthracene. J. Chem. Phys. 63, 4187-4194 (1975).

25. Hu, B. \& Wu, Y. Tuning magnetoresistance between positive and negative values in organic semiconductors. Nat. Mater. 6, 985-991 (2007). 
26. Mihailetchi, V. D., Koster, L. J. A., Hummelen, J. C. \& Blom, P. W. M. Photocurrent generation in polymer-fullerene bulk heterojunctions. Phys. Rev. Lett. 93, 216601 (2004).

27. Clarke, T. M. \& Durrant, J. R. Charge Photogeneration in organic solar cells. Chem. Rev. 110, 6736-6767 (2010).

28. Bernardi, M. et al. Nanocarbon-based photovoltaics. ACS Nano 6, 8896-8903 (2012).

29. Onsager, L. Initial recombination of ions. Phys. Rev. 54, 554-557 (1938).

30. Kataura, H. et al. Dielectric constants of $\mathrm{C}_{60}$ and $\mathrm{C}_{70}$ thin films. J. Phys. Chem. Solids 58, 1913-1917 (1997).

31. Araujo, P. T., Jorio, A., Dresselhaus, M. S., Sato, K. \& Saito, R. Diameter dependence of the dielectric constant for the excitonic transition energy of singlewall carbon nanotubes. Phys. Rev. Lett. 103, 146802 (2009).

32. Green, A. A. \& Hersam, M. C. Nearly single-chirality single-walled carbon nanotubes produced via orthogonal iterative density gradient ultracentrifugation. Adv. Mater. 23, 2185-2190 (2011).

33. Shastry, T. A., Morris-Cohen, A. J., Weiss, E. A. \& Hersam, M. C. Probing carbon nanotube-surfactant interactions with two-dimensional DOSY NMR. J. Am. Chem. Soc. 135, 6750-6753 (2013).

\section{Acknowledgments}

S.R. thanks the financial support from the Army Research Office - Young Investigator Award (W911NF-14-1-0443) for nanocarbon self-assembly and optoelectronics, and US Department of Energy award (DE-FG02-13ER46937) for organic magnetic study. M.C.H. acknowledges funding from the National Science Foundation (DMR-1006391). T.S. is supported by a National Science Foundation Graduate Research Fellowship and the ANSER
Center, an Energy Frontier Research Center funded by the U.S. Department of Energy, Office of Science, Office of Basic Energy Sciences, under Award Number DE-SC0001059.

\section{Author contributions}

W.Q. did the experimental measurements. S.R. guided the research. S.R. and W.Q. prepared manuscript. M.G. took the TEM images. T.S. and M.C.H. investigated the SWCNT materials, and contributed to the manuscript. All authors contributed to data analysis and discussions.

\section{Additional information}

Supplementary information accompanies this paper at http://www.nature.com/ scientificreports

Competing financial interests: The authors declare no competing financial interests.

How to cite this article: Qin, W., Gong, M., Shastry, T., Hersam, M.C. \& Ren, S.

Charge-Transfer Induced Magnetic Field Effects of Nano-Carbon Heterojunctions. Sci. Rep. 4, 6126; DOI:10.1038/srep06126 (2014)

This work is licensed under a Creative Commons Attribution-NonCommercialShareAlike 4.0 International License. The images or other third party material in this article are included in the article's Creative Commons license, unless indicated otherwise in the credit line; if the material is not included under the Creative Commons license, users will need to obtain permission from the license holder in order to reproduce the material. To view a copy of this license, visit http:// creativecommons.org/licenses/by-nc-sa/4.0/ 